\title{
COMPOSIÇÃO FLORÍSTICO-ESTRUTURAL AO LONGO DE UM GRADIENTE DE BORDA EM FRAGMENTO DE FLORESTA OMBRÓFILA MISTA ALTO-MONTANA EM SANTA CATARINA
}

\section{FLORISTIC-STRUCTURAL COMPOSITION ALONG AN EDGE GRADIENT IN UPPER MONTANE MIXED OMBROPHILOUS FOREST FRAGMENT IN SANTA CATARINA}

\author{
Tiago de Souza Ferreira ${ }^{1}$ Amanda Koche Marcon ${ }^{1}$ Bruna Salami ${ }^{1}$ Carla Carolina Chini Rech ${ }^{2}$ \\ Alex Ribeiro Mendes ${ }^{2}$ Adriel Furtado Carvalho ${ }^{3}$ Francieli de Fátima Missio ${ }^{4}$ Francieli Pscheidt $^{4}$ \\ André Luiz Guidini ${ }^{1}$ Rafael da Silva Dornelles ${ }^{4}$ Ana Carolina Silva ${ }^{5}$ Pedro Higuchi ${ }^{6}$
}

\section{RESUMO}

O objetivo desse estudo foi caracterizar a composição florística e a estrutura do componente arbóreo em fragmento de Floresta Ombrófila Mista Alto-Montana e avaliar a influência do efeito de borda sobre a organização, estrutura, riqueza e diversidade de espécies. Foram alocadas 50 parcelas permanentes de 10 × 20 m, divididas em cinco transeções distanciadas, no mínimo, $100 \mathrm{~m}$ entre si, em um fragmento florestal, no município de Bom Jardim da Serra - SC. As árvores com circunferência $\geq 15,7 \mathrm{~cm}$ na altura do peito (CAP) foram mensuradas (CAP e altura total), identificadas e classificadas quanto às guildas de regeneração (pioneiras, climácicas exigentes em luz e climácicas tolerantes à sombra). Os dados foram analisados por meio dos índices de valor de importância (IVI), NMDS (Nonmetric Multidimensional Scaling), modelo aditivo generalizado e regressões lineares simples. Foram observados 1.457 indivíduos arbóreos, distribuídos em 29 famílias, 43 gêneros e 55 espécies. A espécie com maior valor de importância foi Dicksonia sellowiana Hook. Não foi observada influência do efeito de borda sobre a organização, a estrutura (diâmetro médio, altura média e densidade) da comunidade e participação relativa das guildas de regeneração. No entanto, ficaram evidenciados maiores valores de diversidade, riqueza e equabilidade nas áreas de borda. Desta forma, concluí-se que parte das variações dos valores relativos à diversidade de espécies arbóreas na Floresta Ombrófila Mista Ato-Montana foi determinada pela distância da borda.

Palavras-chave: Floresta de Araucária; efeito de borda; diversidade biológica.

1 Engenheiro Florestal, MSc., Departamento de Engenharia Florestal, Centro de Ciências Agroveterinárias, Universidade do Estado de Santa Catarina, Av. Luis de Camões, 2090, CEP 88520-000, Lages (SC), Brasil. tiagoferreira@florestal.eng.br / amandamarcon@yahoo.com.br / brunaflorestal@yahoo.com.br/ andrelg85@yahoo.com.br

2 Bióloga, Departamento de Engenharia Florestal, Centro de Ciências Agroveterinárias, Universidade do Estado de Santa Catarina, Av. Luis de Camões, 2090, CEP 88520-000, Lages (SC), Brasil. karol_chini@hotmail.com / alexrmbio@yahoo.com.br

3 Engenheiro Agrônomo, MSc., Departamento de Agronomia, Centro de Ciências Agroveterinárias, Universidade do Estado de Santa Catarina, Av. Luis de Camões, 2090, CEP 88520-000, Lages (SC), Brasil. adrielfurtado@yahoo.com.br

4 Engenheira Florestal, Departamento de Engenharia Florestal, Centro de Ciências Agroveterinárias, Universidade do Estado de Santa Catarina, Av. Luis de Camões, 2090, CEP 88520-000, Lages (SC), Brasil. franmissio@yahoo. com.br / francieli_pscheidt@hotmail.com/rsdflorestal@yahoo.com.br

5 Engenheira Florestal, Dra ${ }^{\text {., }}$ Professora Adjunto do Departamento de Engenharia Florestal, Centro de Ciências Agroveterinárias, Universidade do Estado de Santa Catarina, Av. Luis de Camões, 2090, CEP 88520-000, Lages (SC), Brasil. carol_sil4@yahoo.com.br

6 Engenheiro Florestal, Dr., Professor Associado do Departamento de Engenharia Florestal, Centro de Ciências Agroveterinárias, Universidade do Estado de Santa Catarina, Av. Luis de Camões, 2090, CEP 88520-000, Lages (SC), Brasil. higuchip@gmail.com

Recebido para publicação em 17/04/2013 e aceito em 8/01/2014 


\begin{abstract}
The objectives of this study were to characterize the floristic and structure of the tree component in Upper Montane Araucaria Forest fragment and to evaluate the influence of the edge effects on tree species organization, structure, richness and diversity. For this, a total of 50, $10 \times 20 \mathrm{~m}$, permanent plots divided in five transects spaced, at least, $100 \mathrm{~m}$ from each other, were established in the a forest fragment, located in the municipality of Bom Jardim da Serra, SC. The trees with circumference at breast height $(\mathrm{CBH}) \geq$ $15,7 \mathrm{~cm}$ were measured ( $\mathrm{CBH}$ and total height), identified and classified according to the regeneration guilds. The data were analyzed through the index of importance values (IVI), a NMDS analysis (Nonmetric Multidimensional Scaling), a generalized additive model and simple linear regressions. A total of 1,457 individuals, distributed in 29 families, 43 genera and 55 species were surveyed. The most relative important species was Dicksonia sellowiana Hook. There was no influence of edge effect on tree community organization, structure (average diameter, average height and density) and guilds participation. However, values of diversity, richness and evenness were higher at edge areas. We conclude that part of the variation in values related to tree species diversity in the Upper Montane Araucaria Forest was determined by edge distance.
\end{abstract}

Keywords: araucaria forest; edge effect; biological diversity.

\section{INTRODUÇÃO}

A Floresta Ombrófila Mista (FOM), também conhecida como "Mata-de-Araucária" (IBGE, 2012), faz parte do complexo vegetacional da Mata Atlântica (OLIVEIRA-FILHO e FONTES, 2000), sendo distribuída, principalmente, no Brasil meridional, entre as latitudes 24 e $30^{\circ} \mathrm{S}$, em altitude que varia de 500 a $1.400 \mathrm{~m}$. Ocorre também na província de Missiones (Argentina), no sul do estado de São Paulo e na Serra da Mantiqueira (KLEIN, 1960). Essa fitofisionomia tem sido considerada uma das mais notáveis em termos de valor ecológico, por abrigar espécies típicas e atributos biológicos únicos em todo o planeta (OLIVEIRA-FILHO et al., 2013).

Atualmente, a conservação das áreas de FOM representa grande desafio para órgãos e entidades ligadas ao meio ambiente, em função do elevado nível de perturbações antrópicas das florestas, sendo que a fragmentação de habitat é uma das principais consequências dessas perturbações (VIANA e PINHEIRO, 1998). A fragmentação age reduzindo áreas e isolando populações (HERRMANN et al., 2005) e, segundo Metzger (2000), implica o aumento nas áreas de bordas em uma paisagem, além de influenciar a composição e a diversidade de espécies na comunidade.

As bordas podem ser definidas como a região de contato entre a matriz antrópica e o fragmento de vegetação natural (WILLIAMSLINERA et al., 1998; PRIMACK e RODRIGUES,
2001). A maior presença deste tipo de área resulta em elevado efeito borda, que são modificações nas áreas mais externas dos fragmentos florestais, geradas pelo contato com a matriz não florestal (MURCIA, 1995), promovendo alterações nos parâmetros físicos, químicos e biológicos do ecossistema. Segundo Rodrigues (1998), os efeitos de borda podem ser divididos em três tipos: abióticos, bióticos diretos e bióticos indiretos. Os efeitos abióticos mais importantes são o aumento da radiação solar, a diminuição da umidade e o aumento da entrada de ventos. Os efeitos biológicos diretos estão relacionados com as alterações causadas pelos efeitos abióticos na comunidade (e.g. aumento da densidade de plantas em função do incremento na radiação solar). Por sua vez, os efeitos bióticos indiretos envolvem mudanças na interação entre as espécies, como predação, herbivoria, dispersão de sementes e polinização.

Em regiões tropicais no Brasil, dentre as principais mudanças florísticas estruturais que ocorrem em função da criação de bordas destaca-se a substituição de espécies tolerantes ao sombreamento por aquelas com maior exigência em luz (e.g., LAURANCE et al., 2002; PUTZ, et al., 2011) nas áreas próximas da matriz não florestal. Estas modificações ocorrem porque a distribuição das espécies em um ambiente reflete o nível de adaptabilidade frente às diversas pressões seletivas atuantes, uma vez que devem existir condições essenciais à sobrevivência para que ocorra a ocupação e a colonização de determinado local 
(RIDLEY, 2006; RIZZINI, 1997). Dessa forma, é possível haver diferenciação na utilização de bordas por espécies ou as bordas podem ter propriedades seletivas, inibindo a dispersão de algumas espécies e facilitando a de outras, podendo resultar em composição florístico-estrutural distinta da área de interior do fragmento. No entanto, apesar destes padrões serem frequentemente observados em regiões tropicais, pouco se conhece sobre o efeito de borda em áreas subtropicais.

As áreas de FOM de Santa Catarina têm sido estudadas por alguns autores (e.g. FORMENTO et al., 2004; ESKUCHE, 2007; KLAUBERG et al., 2010; NASCIMENTO et al., 2011; AGUIAR et al., 2012; HIGUCHI et al., 2012a; HIGUCHI et al., 2012b; SILVA et al., 2012; HIGUCHI et al., 2013), porém, são raros os estudos que investiguem o efeito da borda sobre a composição florísticoestrutural dessas florestas (e.g., FONTOURA et al., 2006). Considerando que a análise da influência do efeito de borda sobre a organização de comunidades de espécies arbóreas é de fundamental importância para o entendimento da ecologia em fragmentos florestais, os objetivos do presente trabalho foram: i) caracterizar a composição florística-estrutural do componente arbóreo de um fragmento de Floresta Ombrófila Mista Alto-Montana no Planalto Sul Catarinense e ii) avaliar a influência do efeito de borda sobre a organização, estrutura, riqueza e diversidade de espécies arbóreas na comunidade avaliada.

\section{MATERIAIS E MÉTODOS}

$\mathrm{O}$ estudo foi realizado em um fragmento de floresta de aproximadamente 250 ha localizado no município de Bom Jardim da Serra, na região do Planalto Sul-Catarinense (Figura 1), na latitude $28^{\circ} 20^{\prime} 30^{\prime \prime}$ S e longitude $49^{\circ} 44^{\prime} 33^{\prime \prime} \mathrm{O}$, com altitude em torno de $1.300 \mathrm{~m}$. O clima predominante é $\mathrm{Cfb}$, de acordo com a classificação de Köppen. A área encontra-se inserida na Bacia Hidrográfica do Rio Pelotas, com topografia de suave-ondulada a ondulada, sendo a vegetação classificada como Floresta Ombrófila Mista Alto-Montana (IBGE, 2012). O fragmento possui grande heterogeneidade ambiental, com a ocorrência de nascentes, riachos e variações topográficas, apresentando áreas de baixada, encostas, grotas e topos de morros. O sítio amostrado está localizado em propriedade rural particular e inserido em uma matriz antropizada, utilizada para fins de agropecuária.
Para o levantamento florístico e da estrutura do componente arbóreo, foram alocadas 50 parcelas permanentes de $10 \times 20 \mathrm{~m}\left(200 \mathrm{~m}^{2}\right)$, divididas em cinco transeções com 10 parcelas cada, totalizando 1 ha de área amostrada. As parcelas foram distribuídas ao longo de cada transeção de forma contínua, sendo as transeções alocadas obedecendo a distância mínima de $100 \mathrm{~m}$ entre si. A primeira parcela de cada transeção foi estabelecida junto à borda do fragmento, sendo que o direcionamento das transeções foi estabelecido com o auxílio de bússola e trena, no sentido do interior do fragmento. Em cada parcela, todos os indivíduos arbóreos vivos que apresentaram CAP (circunferência a altura do peito, medida a $1,30 \mathrm{~m}$ do solo) igual ou superior a $15,7 \mathrm{~cm}$ foram identificados, tiveram o CAP medido com fita métrica, a altura estimada com auxílio de um podão com altura conhecida e marcados com plaquetas de alumínio. Indivíduos com troncos múltiplos foram medidos quando a raiz quadrada da soma dos quadrados dos CAPs foi maior ou igual a $15,7 \mathrm{~cm}$. As identificações foram realizadas por meio de literatura e consulta a especialistas. As espécies foram classificadas de acordo com o sistema Angiosperm Phylogeny Group - APG III (APG, 2009).

Com o objetivo de verificar a eficácia da amostragem, foi gerada curva de acumulação de espécies, com base no método de rarefação (HELTSHE e FORRESTER, 1983). Para a contextualização regional da riqueza utilizouse a técnica de rarefação (HECK et al., 1975). Para a quantificação da diversidade de espécies arbóreas e dominância ecológica, foram calculados, respectivamente, o índice de diversidade de Shannon (H') e o índice de equabilidade de Pielou (J') (BROWER et al., 1990).

A estrutura da comunidade arbórea foi descrita a partir do cálculo, para cada espécie, dos descritores quantitativos clássicos propostos por Mueller-Dombois e Ellemberg (1974). Também foi realizada a classificação das espécies arbóreas em guildas de regeneração, utilizandose as classes definidas por Swaine e Whitmore (1988), que dividiram as espécies em dois grandes grupos: pioneiras, aquelas que necessitam de luz para germinar e completar todo o ciclo de vida, e climácicas, aquelas que frequentemente ocorrem no sub-bosque florestal. Conforme Oliveira-Filho et al. (1994), o grupo das climácicas foi subdivido em função da exigência lumínica das espécies em "clímax exigentes em luz" (CEL), aquelas que 


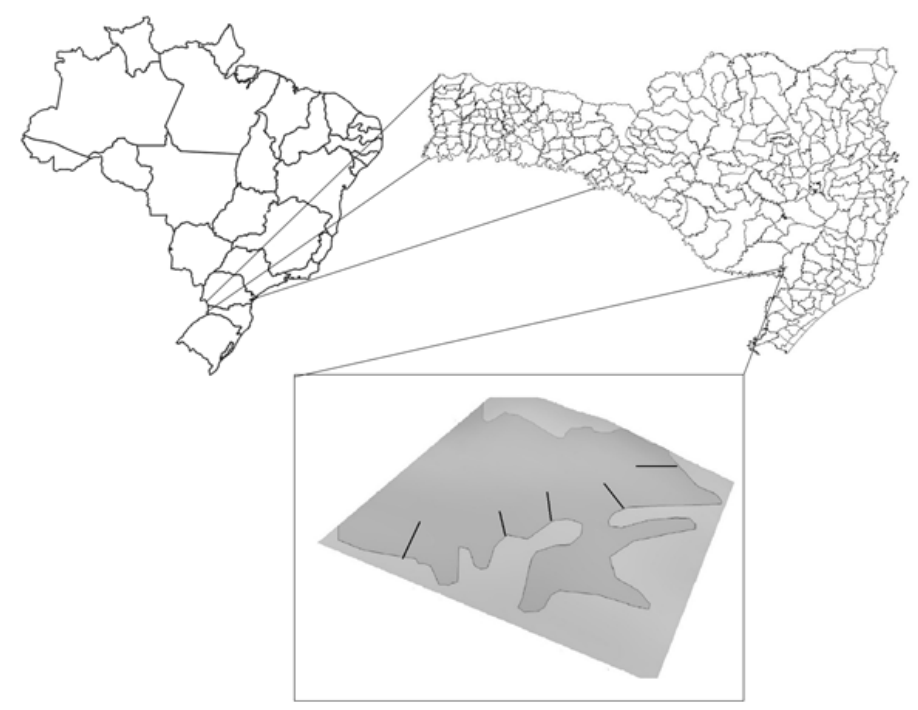

FIGURA 1: Localização da área de estudo com indicação das transeções amostrais de 20 x $100 \mathrm{~m}$, perpendiculares às bordas, em fragmento de Floresta Ombrófila Mista Alto-Montana, no município de Bom Jardim da Serra - SC.

FIGURE 1: Study area location with indication of $20 \mathrm{x} 100 \mathrm{~m}$ sampling transects, perpendicular to the edges, in Upper-Montane Araucaria Forest fragment in the municipality of Bom Jardim da Serra, SC.

precisam de luz para completar o ciclo de vida, e "clímax tolerantes ao sombreamento" (CTS), aquelas que germinam em condição de sub-bosque e podem completar todo o ciclo de vida nestas condições. Para a classificação das espécies foi realizada consultas à literatura (LINDENMAIER e BUDKE, 2006; LEYSER et al., 2012) e observações pessoais dos autores no campo.

Por meio do programa $\mathrm{R}$ ( $\mathrm{R}$ DEVELOPMENT CORE TEAM, 2012), utilizando o pacote Vegan (OKSANEN et al., 2009), foi realizada a ordenação das parcelas em função das características estruturais (abundância) e florísticas, por meio de uma matriz de dados quantitativos, utilizando a técnica de análise multivariada NMDS (Nonmetric Multidimensional Scaling), empregando três dimensões $(\mathrm{k}=3)$ (MINCHIN, 1987). A adequabilidade da ordenação para interpretação foi avaliada por meio do valor de STRESS (Standard Residuals Sum of Squares) (OKSANEN, 2013). O ajuste entre a distância da borda e a ordenação produzida foi realizado a posteriori por meio da função ordsurf, utilizando modelo aditivo generalizado, com thin plate splines (WOOD, 2003). Para verificar a existência de correlações entre o componente arbóreo e a distância da borda, foram utilizadas regressões lineares simples, tendo como variável independente a distância da borda e variáveis dependentes, os valores encontrados por parcela referente às características estruturais (diâmetro médio, altura média e densidade média), estágio sucessional (porcentagens de espécies pioneiras, climácicas exigentes de luz e climácicas tolerantes à sombra) e diversidade (riqueza, índice de diversidade de Shannon e equabilidade de Pielou).

\section{RESULTADOS E DISCUSSÃO}

\section{Florística e estrutura}

Foram amostrados 1.457 indivíduos arbóreos distribuídos em 29 famílias, 43 gêneros e 55 espécies (Tabela 1), sendo que um indivíduo não pode ser identificado devido à ausência de material botânico vegetativo e/ou reprodutivo, durante a realização do inventário. A riqueza de espécies encontrada (55 espécies) pode ser considerada baixa quando comparada com FOM Montana, com ocorrência em faixa altitudinal inferior a $1.000 \mathrm{~m}$ (e.g. HIGUCHI et al., 2012b em Lages, com 92 espécies e SILVA et al., 2012, em Lages, com 87 espécies), semelhante a outra FOM AltoMontana, presente na mesma faixa altitudinal (em 
torno de 1.300 m) (e.g. HIGUCHI et al., 2013, em Painel com 50 espécies). Considerando a mesma intensidade amostral entre estas áreas $(\mathrm{n}=1.395$ indivíduos, referente à área em Painel), por meio da técnica de rarefação, observa-se que o padrão foi o mesmo. O valor de diversidade pode ser considerado intermediário $\left(\mathrm{H}^{\prime}=3,21\right)$, considerando as mesmas áreas: Higuchi et al. (2012b) e Silva et al. (2012) que encontraram $\mathrm{H}^{\prime}=3,74$ e 3,60, respectivamente, em Lages; e Higuchi et al. (2013), que encontraram H' $=2,79$ em Painel. Além disso, foi encontrado valor de equabilidade de Pielou (J) de 0,79, o que indica baixa dominância ecológica, com distribuição relativamente homogênea entre as espécies.

Apesar da riqueza de espécies não muito expressiva, quando comparada com outras FOM em altitudes menores, a curva de acumulação de espécies (Figura 2) demonstrou que a amostragem foi adequada para a representação florística da área, uma vez que a inclusão da última parcela resultou em acréscimo de apenas $0,27 \%$ na riqueza observada de espécies. De acordo com Kersten e Galvão (2011), atinge-se a suficiência quando a linha tende à estabilidade e a adição de novas espécies não altera significativamente o número de

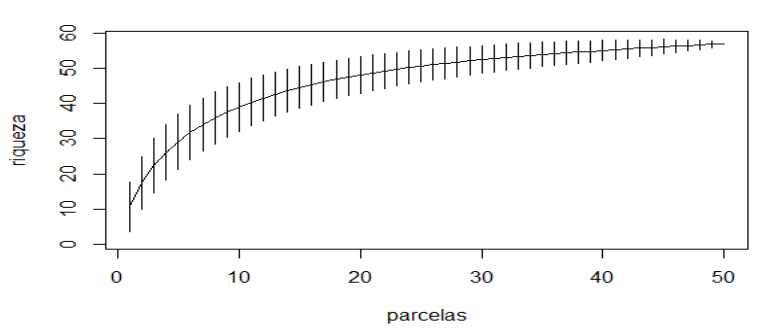

FIGURA 2: Curva de acumulação de espécies arbóreas $(\mathrm{CAP} \geq 15,7 \mathrm{~cm})$ amostradas em fragmento de Floresta Ombrófila Mista Alto-Montana em Bom Jardim da Serra, Santa Catarina. As barras verticais representam os limites de confiança do número de espécies em cada parcela, com base no método de rarefação.

FIGURE 2: Accumulation curve (cbh $>\geq 15.7$ $\mathrm{cm}$ ) of species sampled in upper montane araucaria forest fragment in the municipality of Bom Jardim da Serra, Santa Catarina State. Vertical bars indicate the confidence limits of number of species sampled in each plot, based on rarefaction method. espécies observadas.

Observando a riqueza por famílias, destacase Myrtaceae (10 espécies), Asteraceae (cinco), Anacardiaceae (quatro) e Lauraceae (quatro), representando cerca de $40 \%$ do total de espécies amostradas. Myrtaceae, com cerca de $18 \%$ do total de espécies encontradas, confirma o padrão encontrado de família de alta riqueza nas áreas de FOM, como observado também nos estudos de Jarenkow e Batista (1987), Nascimento et al. (2001), Seger et al. (2005), Kozera et al. (2006), Lingner et al. (2007), Higuchi et al. (2012a), Higuchi et al. (2012b) e Silva et al. (2012). Os gêneros de maior riqueza foram Myrceugenia (quatro), Schinus (três), Ilex (dois), Baccharis (dois), Maytenus (dois), Myrcia (dois), Zanthoxylum (dois), Xylosma (dois) e Solanum (dois). Myrceugenia (Myrtaceae) é frequentemente citado como de elevada riqueza em FOM Alto-Montana, conforme demonstrado no estudo de Higuchi et al. (2013).

A densidade de indivíduos (1.457 ind./ha) foi inferior ao valor encontrado por Higuchi et al. (2012b) (1.843 ind./ha) e por Silva et al. (2012) (1.798 ind./ha) em FOM Montana, no município de Lages, e semelhante aos valores encontradas em FOM Alto-Montana no município de Painel (1.395 ind./ha) (HIGUCHI et al., 2013). Isso sugere tendência a uma menor densidade arbórea em FOMs de maior altitude na região, o que é contrário ao que a literatura indica em outras fitofisionomias (e.g. MEIRELES et al., 2008; SCHEER et al., 2011).

Os indivíduos amostrados totalizaram área basal de $44,49 \mathrm{~m}^{2}$ (Tabela 2), que representa o valor mais elevado já registrado entre os fragmentos de FOM amostrados na região, considerando o mesmo nível de inclusão e em diferentes faixas altitudinais. No município de Lages, em florestas Montanas, Silva et al. (2012) e Higuchi et al. (2102b) encontraram, respectivamente, $35,90 \mathrm{~m}^{2} / \mathrm{ha} 36,45 \mathrm{~m}^{2} / \mathrm{ha}$. Em área de FOM Alto-Montana no município de Painel, Higuchi et al. (2013) encontraram área basal de $34,80 \mathrm{~m}^{2} /$ ha.

Das espécies com os maiores valores de importância (VI), destacaram-se Dicksonia sellowiana Hook., Lithraea brasiliensis Marchand e Araucaria angustifolia (Bertol.) Kuntze. (Tabela 2), que representaram $32 \%$ do VI, e frequentemente são observadas como espécies comuns em fragmentos florestais na região (NASCIMENTO et al., 2001; RONDON NETO et al., 2002; FORMENTO et al., 2004; SEGER et al., 2005; VIBRANS et al., 2011; HIGUCHI et al., 2012a; SILVA et al., 2012). As 
TABELA 1: Estimadores fitossociológicos das espécies arbóreas (CAP $\geq 15,7 \mathrm{~cm}$ ) amostradas em fragmento de Floresta Ombrófila Mista Alto-Montana, Bom Jardim da Serra, Santa Catarina. As espécies estão ordenadas de forma decrescente pelo valor de importância (VI, em \%).

TABLE 1: Phytosociological estimative of species $(\mathrm{cbh} \geq 15.7 \mathrm{~cm})$ sampled in upper montane araucaria forest fragment in the municipality of Bom Jardim da Serra, SC. The species are sorted by descending order of importance value (VI, in \%).

\begin{tabular}{|c|c|c|c|c|c|c|c|}
\hline Espécie & DA & DR & DOA & DOR & FA & FR & VI \\
\hline Dicksonia sellowiana & 167 & 11,46 & 11,19 & 25,16 & 32 & 3,03 & 13,22 \\
\hline Lithraea brasiliensis & 176 & 12,08 & 4,76 & 10,70 & 48 & 4,55 & 9,11 \\
\hline Araucaria angustifolia & 97 & 6,66 & 6,24 & 14,03 & 66 & 6,25 & 8,98 \\
\hline Podocarpus lambertii & 125 & 8,58 & 3,87 & 8,70 & 60 & 5,68 & 7,66 \\
\hline Blepharocalyx salicifolius & 97 & 6,66 & 3,22 & 7,24 & 66 & 6,25 & 6,71 \\
\hline Acca sellowiana & 96 & 6,59 & 0,95 & 2,13 & 76 & 7,20 & 5,30 \\
\hline Scutia buxifolia & 68 & 4,67 & 1,20 & 2,71 & 62 & 5,87 & 4,42 \\
\hline Myrceugenia euosma & 66 & 4,53 & 0,82 & 1,84 & 44 & 4,17 & 3,51 \\
\hline Sebastiania commersoniana & 70 & 4,80 & 1,43 & 3,22 & 20 & 1,89 & 3,31 \\
\hline Ocotea pulchella & 29 & 1,99 & 0,78 & 1,76 & 36 & 3,41 & 2,39 \\
\hline Myrsine coriaea & 25 & 1,72 & 1,12 & 2,51 & 26 & 2,46 & 2,23 \\
\hline Schinus lentiscifolius & 33 & 2,26 & 0,96 & 2,15 & 24 & 2,27 & 2,23 \\
\hline Zanthoxylum rhoifolium & 27 & 1,85 & 0,70 & 1,56 & 34 & 3,22 & 2,21 \\
\hline Symplocos uniflora & 25 & 1,72 & 0,52 & 1,16 & 34 & 3,22 & 2,03 \\
\hline Citronella gongonha & 22 & 1,51 & 0,33 & 0,75 & 36 & 3,41 & 1,89 \\
\hline Myrrhinium atropurpureum & 34 & 2,33 & 0,28 & 0,62 & 28 & 2,65 & 1,87 \\
\hline Allophylus edulis & 25 & 1,72 & 0,26 & 0,58 & 32 & 3,03 & 1,78 \\
\hline Mimosa scabrella & 13 & 0,89 & 1,05 & 2,37 & 16 & 1,52 & 1,59 \\
\hline Colletia paradoxa & 32 & 2,20 & 0,21 & 0,46 & 16 & 1,52 & 1,39 \\
\hline Cinnamomum amoеnum & 11 & 0,75 & 0,61 & 1,38 & 20 & 1,89 & 1,34 \\
\hline Quillaja brasiliensis & 7 & 0,48 & 1,04 & 2,34 & 12 & 1,14 & 1,32 \\
\hline Drimys brasiliensis & 15 & 1,03 & 0,16 & 0,35 & 24 & 2,27 & 1,22 \\
\hline Myrceugenia ovata & 17 & 1,17 & 0,11 & 0,25 & 20 & 1,89 & 1,10 \\
\hline Symphyopappus itatiayensis & 17 & 1,17 & 0,59 & 1,34 & 8 & 0,76 & 1,09 \\
\hline Myrceugenia sp. & 17 & 1,17 & 0,14 & 0,31 & 14 & 1,33 & 0,93 \\
\hline Baccharis semiserrata & 20 & 1,37 & 0,08 & 0,17 & 10 & 0,95 & 0,83 \\
\hline Myrcia oligantha & 14 & 0,96 & 0,11 & 0,25 & 12 & 1,14 & 0,78 \\
\hline Ilex microdonta & 10 & 0,69 & 0,17 & 0,38 & 12 & 1,14 & 0,74 \\
\hline Rhamnus sphaerosperma & 8 & 0,55 & 0,05 & 0,11 & 16 & 1,52 & 0,72 \\
\hline Maytenus boaria & 7 & 0,48 & 0,24 & 0,53 & 12 & 1,14 & 0,72 \\
\hline Schinus polygamus & 8 & 0,55 & 0,10 & 0,21 & 12 & 1,14 & 0,63 \\
\hline Clethra scabra & 6 & 0,41 & 0,09 & 0,21 & 12 & 1,14 & 0,59 \\
\hline Prunus myrtifolia & 5 & 0,34 & 0,28 & 0,63 & 8 & 0,76 & 0,58 \\
\hline Berberis laurina & 7 & 0,48 & 0,03 & 0,08 & 12 & 1,14 & 0,56 \\
\hline Myrcia palustres & 6 & 0,41 & 0,07 & 0,15 & 8 & 0,76 & 0,44 \\
\hline Xylosma tweediana & 6 & 0,41 & 0,04 & 0,09 & 8 & 0,76 & 0,42 \\
\hline Solanum compressum & 4 & 0,27 & 0,05 & 0,12 & 8 & 0,76 & 0,38 \\
\hline Nectandra megapotamica & 6 & 0,41 & 0,07 & 0,15 & 6 & 0,57 & 0,38 \\
\hline Xylosma ciliatifolia & 4 & 0,27 & 0,02 & 0,05 & 8 & 0,76 & 0,36 \\
\hline Oreopanax fulvum & 4 & 0,27 & 0,01 & 0,02 & 8 & 0,76 & 0,35 \\
\hline Baccharis uncinella & 6 & 0,41 & 0,03 & 0,07 & 6 & 0,57 & 0,35 \\
\hline Weinmannia paulliniifolia & 4 & 0,27 & 0,16 & 0,36 & 4 & 0,38 & 0,34 \\
\hline Solanum pabstii & 3 & 0,21 & 0,06 & 0,14 & 6 & 0,57 & 0,30 \\
\hline Zanthoxylum kleinii & 3 & 0,21 & 0,05 & 0,11 & 6 & 0,57 & 0,30 \\
\hline Schinus terebinthifolius & 2 & 0,14 & 0,13 & 0,29 & 2 & 0,19 & 0,20 \\
\hline Persea willdenovii & 2 & 0,14 & 0,01 & 0,02 & 4 & 0,38 & 0,18 \\
\hline Piptocarpha angustifolia & 2 & 0,14 & 0,01 & 0,02 & 4 & 0,38 & 0,18 \\
\hline Ilex taubertiana & 1 & 0,07 & 0,02 & 0,05 & 2 & 0,19 & 0,10 \\
\hline Styrax leprosus & 1 & 0,07 & 0,02 & 0,05 & 2 & 0,19 & 0,10 \\
\hline Não identificada & 1 & 0,07 & 0,01 & 0,03 & 2 & 0,19 & 0,09 \\
\hline Maytenus muelleri & 1 & 0,07 & 0,01 & 0,02 & 2 & 0,19 & 0,09 \\
\hline Myrcianthes gigantea & 1 & 0,07 & 0,01 & 0,02 & 2 & 0,19 & 0,09 \\
\hline Celtis brasiliensis & 1 & 0,07 & 0,01 & 0,01 & 2 & 0,19 & 0,09 \\
\hline Myrceugenia miersiana & 1 & 0,07 & 0,01 & 0,01 & 2 & 0,19 & 0,09 \\
\hline Vernonanthura discolor & 1 & 0,07 & 0,00 & 0,01 & 2 & 0,19 & 0,09 \\
\hline Duranta vestita & 1 & 0,07 & 0,00 & 0,01 & 2 & 0,19 & 0,09 \\
\hline Total & 1.457 & 100 & 44,49 & 100 & - & 100 & 100 \\
\hline
\end{tabular}

Em que: DA = Densidade absoluta (ind./ha); DR = densidade relativa (\%); DOA = dominância absoluta ( $\left.\mathrm{m}^{2} / \mathrm{ha}\right)$; $\mathrm{DOR}=$ dominância relativa $(\%) ; \mathrm{FA}=$ frequência absoluta $(\%) ; \mathrm{FR}=$ frequência relativa $(\%)$. 
espécies com menor participação na comunidade, representadas por apenas um indivíduo, como Celtis brasiliensis (Gardner) Planch, Duranta vestita Cham e Vernonanthura discolor (Spreng.) H.Rob, por exemplo, representaram $14 \%$ do total de espécies amostradas. Desta forma, apesar de representarem menos do que $1 \%$ dos indivíduos amostrados e contribuírem pouco para estruturação da floresta, o grupo de espécies raras compõe importante parte da riqueza da comunidade, sendo este um padrão recorrente em vários ecossistemas (MOUILLOT et al., 2013).

\section{Influência da distância da borda sobre os padrões florístico-estruturais}

Não ficaram evidenciados agrupamentos das parcelas em função do setor borda e interior
(Figura 3). O resultado não significativo (variação explicada $=25,2 \%, p=0,268$ ) do ajuste entre borda e a ordenação produzida confirma que a organização estrutural do componente arbóreo não apresentou variações espaciais associadas à distância da borda. Esse resultado contrasta com os observados por Oliveira-Filho et al. (2004), em fragmento de Floresta Estacional Semidecidual Alto-Montana, no Sul de Minas Gerais, que indicaram diferenças abruptas entre a comunidade de árvores da borda e do interior. No entanto, da mesma forma como observado no presente estudo, em alguns fragmentos de diferentes formações florestais, não foi constatada a influência da distância da borda sobre os parâmetros florísticoestruturais da comunidade vegetal arbórea (e.g. PHILLIPS et al., 2006; MULLER et al., 2010; SAMPAIO e SCARIOT, 2011).

Apesar de não ser evidenciada influência da

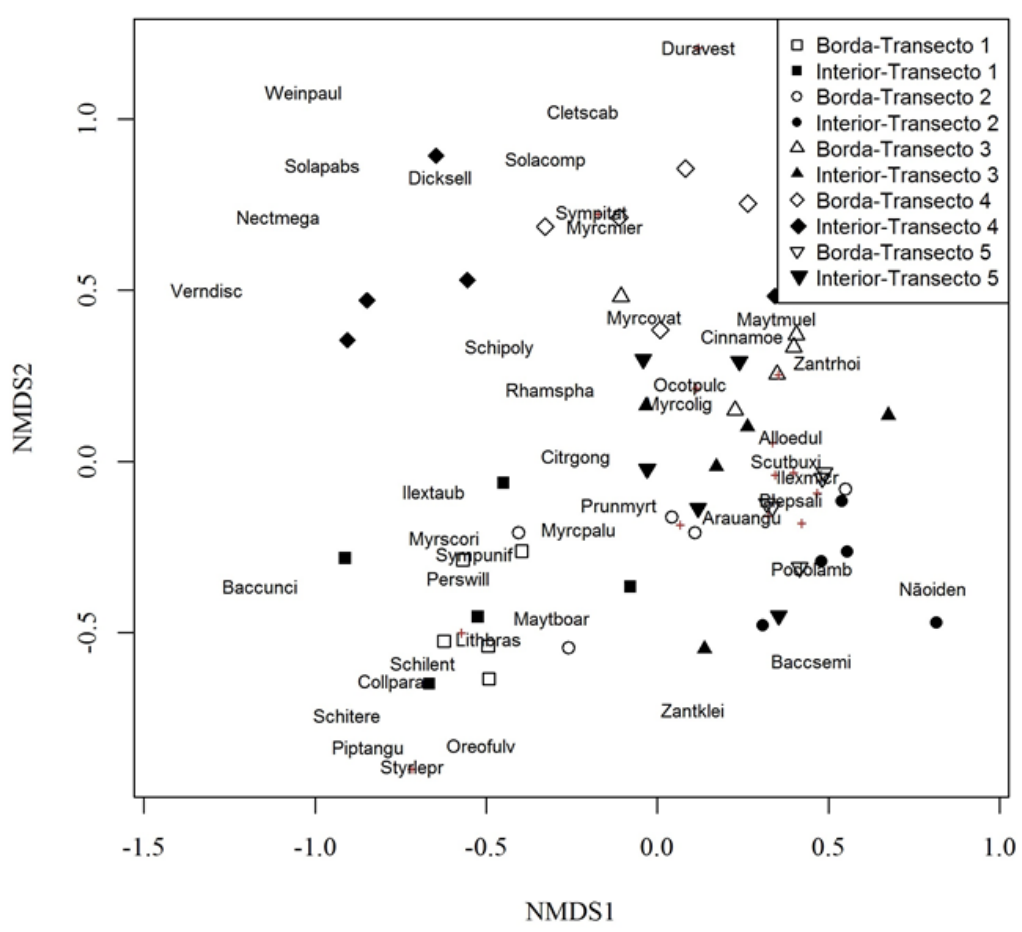

FIGURA 3: Diagrama de ordenação produzido pela análise NMDS (Nonmetric multidimensional scalling) (valor de estresse $=18,91, \mathrm{k}=3$ ) para as parcelas (50 parcelas, $10 \times 20 \mathrm{~m}$ ) e as espécies arbóreas $(\mathrm{CAP} \geq 15,7 \mathrm{~cm})$, amostradas no fragmento estudado de Floresta Ombrófila Mista AltoMontana localizado em Bom Jardim da Serra, Santa Catarina. Símbolos sem preenchimento indicam parcelas de borda e símbolos com preenchimento indicam as parcelas de interior. Diferentes formatos de símbolos indicam as diferentes transeções.

FIGURE 3: Ordination diagram of plots (50 plots, $10 \times 20 \mathrm{~m})$, and tree species $(\mathrm{cbh} \geq 15.7 \mathrm{~cm})$ produced by NMDS analysis (Nonmetric multidimensional scalling) (stress value $=18,91 \%, \mathrm{k}=3$ ), in the studied fragment of Upper-montane Araucaria Forest, located in Bom Jardim da Serra, SC. Open symbols indicate the edge sectors and filled symbols indicate the interior sectors. Differents shapes of symbols indicate different transects. 
borda, os resultados sugerem variações florísticoestruturais entre as transeções, que podem ser explicadas pela heterogeneidade ambiental existente no fragmento. Na Figura 3, esta variação pode ser observada, por exemplo, entre as transeções 1 e 4 , que se situaram em extremos opostos ao longo do eixo 2 da ordenação. A transeção 1, que foi instalada em área de encosta, no terço superior do terreno, teve como espécies associadas: Piptocarpha angustifolia Dusén, Schinus lentiscifolius Marchand, Schinus terebinthifolius Raddi, Lithraea brasiliensis, Baccharis uncinella DC, entre outras. A transeção 4 foi instalada em área de baixada, tendo como espécies representativas: Nectandra megapotamica (Spreng.) Mez, Dicksonia sellowiana, Symphyopappus itatiayensis (Hieron.) R.M.King \& H.Rob, entre outras. A NMDS também demonstrou que ocorreu partição espacial, não associada à distância da borda, das espécies pertencentes ao grupo ecológico das pioneiras, como Baccharis uncinella, Baccharis semiserrata DC, Clethra scabra Pers e Vernonanthura discolor. Este resultado sugere que os trechos em estágio inicial de sucessão no fragmento não são homogêneos em termos de identidade florística. Estas diferenças observadas podem estar relacionadas a diversos gradientes ambientais, como os relacionados aos fatores edáficos e, também, a aspectos relacionados à ecologia e traços funcionais das espécies, como síndromes de dispersão e polinização e exigências quanto ao habitat (MURCIA, 1995; RODRIGUES, 1998; LAURANCE et al., 1998; TABARELLI et al., 2004).

Variáveis estruturais, como o diâmetro (Figura 4A), a altura (Figura 4B) e a densidade (Figura 4C), não foram afetadas pela distância da borda, com valores de $p$ na análise de regressão não significativos. Também não foram encontradas correlações entre a porcentagem de espécies pertencentes aos diferentes grupos sucessionais em função da distância da borda (Figuras 4D, 4E e $4 \mathrm{~F})$. Em relação às variáveis relativas à diversidade, à riqueza (Figura 4G), o índice de diversidade de Shannon (Figura 4H) e a equabilidade de Pielou (Figura 4I) tiveram correlações significativas $(p \leq 0,1)$.

O baixo número de variáveis afetadas pela distância da borda pode ser explicado pelo histórico de perturbação da área, no qual séculos de utilização (e.g. corte seletivo, presença de gado e queimadas) imprimiram mudanças ambientais, estruturais e florística no fragmento. Fenômeno semelhante foi relatado por Muller etal. (2010) ao estudarem o efeito de borda sobre a estrutura da comunidade arbórea em fragmento de FOM no Rio Grande do Sul, pois não encontraram influência da borda sobre a densidade arbórea. No que se refere às mudanças ambientais, destacam-se a presença de trilhas e estradas antigas e maior incidência de clareiras no interior da mata. Estas alterações podem criar condições ambientais no interior de fragmentos semelhantes àquelas observadas nas bordas (e.g. maiores temperaturas, baixa umidade e aumento nos níveis de radiação solar). Isso resulta, em longo prazo, em mudanças na abundância e distribuição das espécies, bem como das interações entre espécies (LAURANCE e BIERREGAARD, 1997; BENITEZ-MALVIDO, 1998; SCARIOT, 1999). Nunes et al. (2003) relataram que a porcentagem elevada de espécies secundárias iniciais na área pode ser explicada por distúrbios ou perturbações ocorridos anteriormente. Assim, destaca-se a possibilidade de ocorrência do processo de homogeneização biológica, representada pela proliferação de espécies pioneiras, adaptadas a distúrbios, como destacado por Lôbo et al. (2011), em fragmentos de Mata Atlântica no Nordeste brasileiro. Os resultados encontrados na classificação sucessional das espécies confirmam o padrão observado no fragmento estudado, pois as espécies de início de sucessão (pioneiras e clímax exigente de luz) compuseram a maioria do componente arbóreo: 51 espécies, correspondendo a $93 \%$, sendo $19 \%$ de pioneiras e $74 \%$ de clímax exigente de luz.

A área de borda do fragmento apresentou maiores valores de riqueza, diversidade e equabilidade do que o interior do mesmo, sendo este o mesmo padrão encontrado por Alves Jr. et al. (2006). Este resultado pode ser explicado, em parte, pelo fato das bordas do fragmento serem zona de contato entre a floresta e a matriz adjacente, podendo, desta forma, receber propágulos de fragmentos próximos, contribuindo para um aumento na diversidade desses locais. Outro fator que poderia explicar a maior diversidade seria uma suposta maior heterogeneidade ambiental nas bordas.

\section{CONCLUSÕES}

O perfil florístico do componente arbóreo avaliado foi caracterizado pelo baixo número de espécies e pela elevada riqueza da família Myrtaceae. Dicksonia sellowiana, Lithraea brasiliensis e 


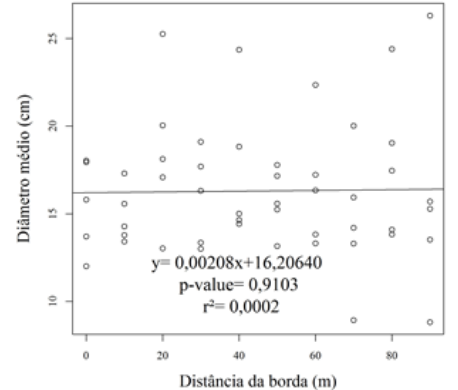

A

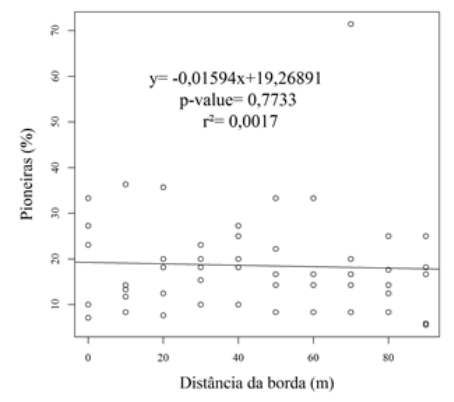

$\mathrm{D}$

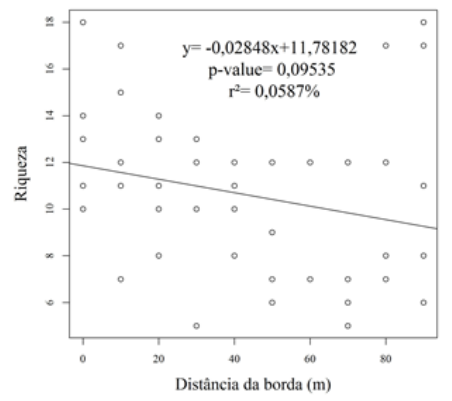

G

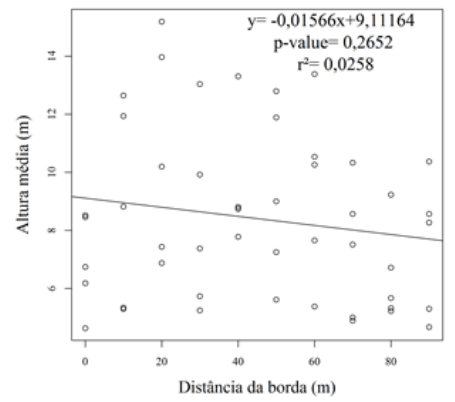

B
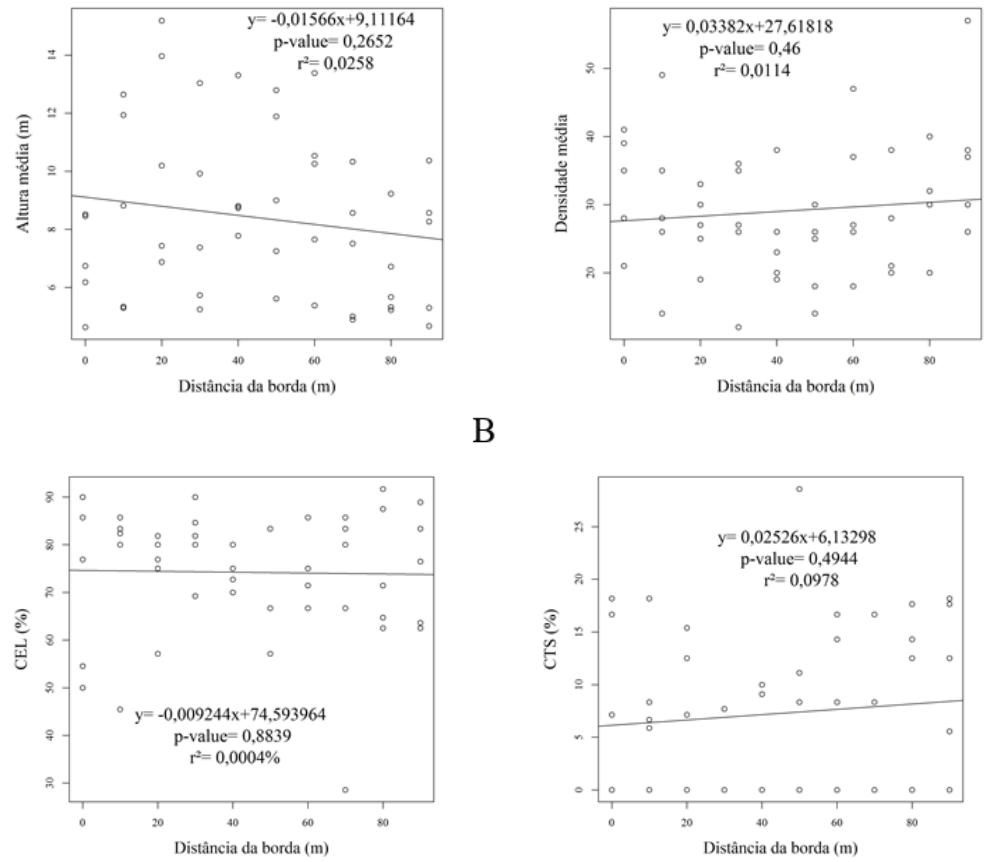

$\mathrm{E}$

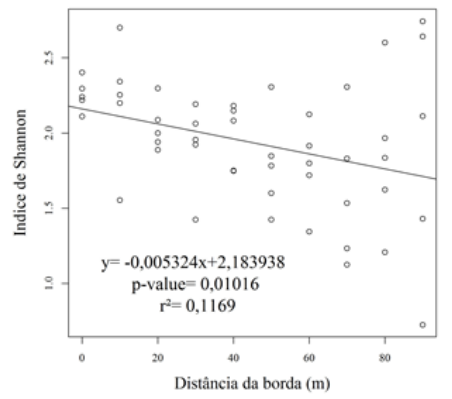

$\mathrm{H}$

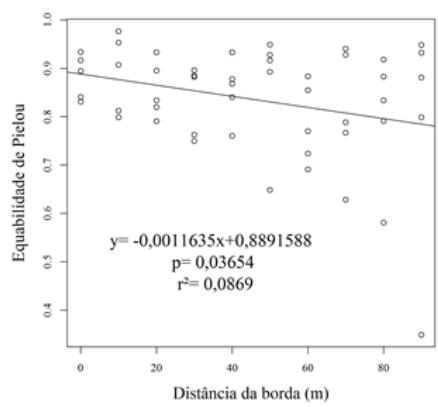

FIGURA 4: Regressão linear entre a distância da borda e os estimadores estruturais [diâmetro médio por parcela (A), altura média por parcela (B) e densidade média por parcela (C)], a classificação sucessional [percentual de espécies pioneiras (D), percentual de espécies clímax exigentes de luz $(\mathrm{E})$ e percentual de espécies clímax tolerantes à sombra $(\mathrm{F})]$, a riqueza $(\mathrm{G})$, o índice de diversidade de Shannon $(\mathrm{H})$ e o índice de equabilidade de Pielou (I) para as espécies arbóreas $(\mathrm{CAP} \geq 15,7 \mathrm{~cm})$ amostradas em fragmento de Floresta Ombrófila Mista Alto-Montana, em Bom Jardim da Serra, Santa Catarina.

FIGURE 4: Linear regression between edge distance and structural estimators [average diameter per plot (A), average height per plot (B) and average density per plot (C)], the successional classification [percentage of pioneer species (D), percentage of light demanding climax species (E) and percentage of shade-tolerant climax species $(F)$ ], richness $(G)$, the Shannon diversity index $(\mathrm{H})$ and evenness (I) in upper-montane Araucaria Forest fragment in the municipality of Bom Jardim da Serra, SC.

Araucaria angustifolia, que são frequentemente observadas como espécies comuns em fragmentos da região, também se destacaram na comunidade estudada. Apesar de não ter sido observada influência do efeito de borda sobre a organização da comunidade, a estrutura (diâmetro médio, altura média e densidade) e participação relativa das guildas de regeneração, ficou evidenciado maiores valores de diversidade, riqueza e equabilidade das espécies arbóreas na área de borda do fragmento estudado. Considerando a natureza complexa do efeito de borda, para maior conhecimento de sua influência 
sobre comunidades de espécies arbóreas na região, recomendam-se estudos contemplando maior escala espacial e diferentes condições ambientais, (e.g., relevo, exposição, forma do fragmento e matriz de entorno).

\section{AGRADECIMENTOS}

Ao $\mathrm{CNPq}$ pelo apoio financeiro, por meio do Edital Universal MCT/CNPq 14/2010 - processo 475095/2010-3, e pela concessão de bolsas de produtividade em pesquisa aos penúltimo e último autores. Ao proprietário rural que gentilmente cedeu a área para o desenvolvimento do presente estudo.

\section{REFERÊNCIAS BIBLIOGRÁFICAS}

AGUIAR, M. D. et al. Potencial de uso de espécies arbóreas de uma floresta secundária em Lages, Santa Catarina. Revista de Ciências Agroveterinárias, Lages, v. 11, n. 3, p. 238-247, set./dez. 2012.

ALVES JR, et al. Efeito de borda na estrutura de espécies arbóreas em um fragmento de Floresta Ombrófila Densa, Recife, PE. Revista Brasileira de Ciências Agrárias, Recife, v. 1, n. único, p. 49-56, out.-dez. 2006.

ANGIOSPERM PHYLOGENY GROUP. An update of the Angiosperm Phylogeny Group classification for the orders and families of flowering plants: APG III. Botanical Journal of the Linnean Society, London, v. 161, n. 2, p. 105-121, 2009.

BENITEZ-MALVIDO, J. Impact of forest fragmentation on seedling abundance in a tropical rain forest. Conservation Biology, Boston, v. 12, n. 2, p. 380-389, 1998.

BROWER, J.; ZAR, J.; VON ENDE, C. Field and laboratory methods for general ecology. Dubuque: McGraw-Hill Science, 1990. 161 p.

ESKUCHE, U. El bosque de Araucaria con Podocarpus y los campos de Bom Jardim da Serra, Santa Catarina (Brasil meridional). Boletín de la Sociedad Argentina de Botánica, Córdoba, v. 42, n. 3-4, p. 295-308, jul./dez. 2007.

FONTOURA, S.B.; GANADE, G.; LAROCCA, J. Changes in plant community diversity and composition across an edge between Araucaria forest and pasture in South Brazil. Brazilian Journal of Botany, v. 29, n.1, São Paulo, Jan-Mar, p. 79-91, 2006.

FORMENTO, S. et al. Dinâmica estrutural arbórea de uma Floresta Ombrófila Mista em Campo Belo do Sul, SC. Cerne, Lavras, v. 10, n. 2, p. 196-212, jul./dez. 2004.

HECK, K.L.; van BELLE, G.; SIMBERLOFF, D. Explicit calculation of the rarefaction diversity measurement and the determination of sufficient sample size. Ecology, Ithaca, v. 56, p. 1459-1461, 1975.

HELTSHE, J.; FORRESTER, N. Estimating species richness using the jackknife procedure. Biometrics, Arlington, v. 39, n. 1, p. 1-11. 1983.

HERRMANN, B. C. et al. A paisagem como condicionadora de bordas de fragmentos florestais. Floresta, Curitiba, v. 35, n. 1, p. 13-22, jan./abr. 2005.

HIGUCHI, P. et al. Floristic composition and phytogeography of the tree component of Araucaria Forest fragments in southern Brazil. Brazilian Journal of Botany, São Paulo, v. 35, n. 2, p. 145-157, abr./jun. 2012a.

HIGUCHI, P. et al. Influência de variáveis ambientais sobre o padrão estrutural e florístico do componente arbóreo, em um fragmento de floresta ombrófila mista montana em Lages, SC. Ciência Florestal, Santa Maria, v. 22, n. 1, p. 79-90, jan./mar. 2012b.

HIGUCHI, P. et al. Florística e estrutura do componente arbóreo e análise ambiental de um fragmento de Floresta Ombrófila Mista Altomontana no município de Painel, SC. Ciência Florestal, Santa Maria, v. 23, n. 1, p. 153-164, jan./mar. 2013.

IBGE. Manual técnico da vegetação brasileira. Rio de Janeiro: Fundação Instituto Brasileiro de Geografia e Estatística, 2012. 271 p.

JARENKOW, J. A.; BAPTISTA, L. R. M. Composição florística e estrutura da mata com araucária na Estação Ecológica de Aracuri, Esmeralda, Rio Grande do Sul. Napaea, Porto Alegre, v. 3, p. 9-18. 1987.

KERSTEN, R.A.; GALVÃO, F. Suficiência amostral em inventários florísticos e fitossociológicos. In: FELFILI, J. M. et al. (Eds.). Fitossociologia no Brasil: Métodos e estudos de casos. Viçosa: Editora UFV, 2011. p. 153-176.

KLAUBERG, C. et al. Florística e estrutura de um fragmento de Floresta Ombrófila Mista no Planalto Catarinense. Biotemas, Florianópolis, v. 23, n. 1, p. 35-47, mar. 2010.

KLEIN, R. O aspecto dinâmico do pinheiro brasileiro. Sellowia, Itajaí, v. 12, p. 17-44. 1960.

KOZERA, C. et al. Fitossociologia do componente arbóreo de um fragmento de Floresta Ombrófila Mista Montana, Curitiba, PR, BR. Floresta, 
Curitiba, v. 36, n. 2, p. 225-237, mai./ago. 2006. LAURANCE, W. F.; BIERREGAARD, R. O. Tropical forest remnants ecology management and conservation of fragmented communities. Chicago: Chicago University Press, 1997. 616 p. LAURANCE, W. F. et al. Rain forest fragmentation and the dynamics of Amazonian tree communities. Ecology, Ithaca, v. 79, n. 6, p. 2032-2040, set. 1998. LAURANCE et al. Ecosystem Decay of Amazonian Forest Fragments: a 22-Year Investigation. Conservation Biology, Hoboken, v. 16, n.3, p. 605-618, jun., 2002.

LEYSER, G. et al. Regeneração de espécies arbóreas e relações com o componente adulto em uma floresta estacional no vale do rio Uruguai, Brasil. Acta Botanica Brasilica, Feira de Santana, v. 26, n. 1, p. 74-83, jan./mar. 2012.

LINDENMAIER, D. S.; BUDKE, J. C. Florística, diversidade e distribuição espacial das espécies arbóreas em uma floresta estacional na bacia do rio Jacuí, Sul do Brasil. Pesquisas Botânicas, São Leopoldo, v. s. número, n. 57, p. 193-216, 2006.

LINGNER, D. et al. Caracterização da estrutura e da dinâmica de um remanescente de Floresta com Araucária no Planalto Catarinense. Pesquisa Florestal Brasileira, Colombo, v. s. número, n. 55, p. 55-66, jul./dez. 2007.

LÔBO, D. et al. Forest fragmentation drives Atlantic Forest of northeastern Brazil biotic homogenization. Diversity and Distribution, v. 17, n. 2, p. 287-296, mar. 2011.

MEIRELES, L. D. et al. Variações na composição florística e na estrutura fitossociológica de uma floresta ombrófila densa alto-montana na Serra da Mantiqueira, Monte Verde, MG. Revista Brasileira de Botânica, São Paulo, v. 31, n. 4, out./dez. p. 559-574. 2008.

METZGER, J. P. Tree functional group richness and landscape structure in Brazilian tropical fragmented landscape. Ecological Applications, New York, v. 10, n. 4, p. 1147 - 1161. 2000.

MINCHIN, P. R. An evaluation of the relative robustness of techniques for ecological ordination. Vegetatio, v. 69, n. 1, p. 89-107. 1987.

MOUILLOT, D. et al. Rare species support vulnerable function in high-diversity ecosystem. PLoS Biology, São Francisco, v.11, n.5, p.1-11, 2013.

MUELLER-DOMBOIS, D.; ELLENBERG, $\mathrm{H}$. Aims and of vegetation ecology. New York: John Wiley \& Sons, 1974. 547 p.

MULLER, A. et al. Efeito de borda sobre a comunidade arbórea em um fragmento de Floresta Ombrófila Mista, Rio Grande do Sul, Brasil. Perspectiva, Erechim, v. 34, n. 125, p. 29-39, mar. 2010

MURCIA, C. Edge Effects in fragmented forests: implications for conservation. Trends in Ecology e Evolution, Amsterdam, v. 10, p. 58-62. 1995.

NASCIMENTO, A. R. T. et al. Estrutura e padrões de distribuição espacial de espécies arbóreas em uma amostra de Floresta Ombrófila Mista em Nova Prata, RS. Ciência Florestal, Santa Maria, v. 11, n. 1, p. 105-119, jan./jun. 2001.

NASCIMENTO, A. R. T. et al. Estrutura e classificação de um remanescente de floresta ripária no município de Lages, SC. Ciência Florestal, Santa Maria, v. 21, n. 2, p. 209-218, abr./jun. 2011. NUNES, Y. R. F. et al. Variações da fisionomia da comunidade arbóreos em um fragmento de Floresta Semidecidual em Lavras, MG. Acta Botânica Brasílica, Feira de Santana, v. 17, n. 2, p. 213-229, abr./jun. 2003.

OKSANEN, J. et al. Vegan: community ecology package. $\mathbf{R}$ package version, v. 1, p. 8-8. 2009.

OKSANEN, J. Multivariate Analysis of Ecological Communities in R: vegan tutorial. 43p. 2013. Disponível em http://cc.oulu.fi/ jarioksa/opetus/ metodi/vegantutor.pdf Acesso em 22/10/2013.

OLIVEIRA-FIHO, A. T. et al. Effects of soils and topography on the distribution of tree species in a tropical riverine forest in south-eastern Brazil. Journal of Tropical Ecology, Cambridge, n. 4, v. 10, 483-508, Nov, 1994.

OLIVEIRA-FILHO, A. T. et al. Variações estruturais do compartimento arbóreo de uma floresta semidecídua Alto-Montana na chapada das Perdizes, Carrancas, MG. Revista Brasileira de Botânica, São Paulo, v. 27, n. 2, p. 291-309, abr./ jun. 2004.

OLIVEIRA-FILHO, A. T.; FONTES, M. A. L. Patterns of floristic differentiation among Atlantic forests in south-eastern Brazil, and the influence of climate. Biotropica, Lawrence, v. 32, n. 4, p. 793-810. 2000.

OLIVEIRA-FILHO et al. Delving into the variations in tree species composition and richness across South American subtropical Atlantic and Pampean forests. Journal of Plant Ecology, Oxford, online first, 2013.

PHILLIPS, O. L. et al. Resilience of southwestern Amazon Forests to anthropogenic edge effects. Conservation Biology, Boston, v. 20, n. 6, p. 1698-1710. 2006. 
PRIMACK, R. B.; RODRIGUES, E. Biologia da Conservação. Londrina: Ed. Planta, 2001. 328 p.

R DEVELOPMENT CORE TEAM. R: A language and environment for statistical computing. $R$ Foundation for Statistical Computing, Vienna. Disponível em: <(http://www.R-project.org)> Acesso em: 21 de agosto de 2012.

PUTZ, S.; GROENEVEL, J.; ALVES, L.F.; METZGER, J.P.; HUTH, A. Fragmentation drives tropical forest fragments to early successional states: A modelling study for Brazilian Atlantic forests. Ecological Modelling, Amsterdam, Jun, v. 222, n., 12, p. 1986-1997.

RIDLEY, M. Evolução. 3 ed. Porto Alegre: Artmed, 2006. $752 \mathrm{p}$.

RIZZINI, C. T. Tratado de Fitogeografia do Brasil. São Paulo: Ed. Âmbito Cultural, 1997. 747 p.

RODRIGUES, E. Edge Effects on the regeneration of forest fragments in South Brasil. 1998. 172 f. Thesis (PhD in Biology) - Harvard University, Cambridge, 1998.

RONDON NETO, R. et al. Caracterização florística e estrutural de um fragmento de floresta ombrófila mista em Curitiba, PR, Brasil. Floresta, Curitiba, v. 32, n. 1, p. 3-16, jan./jul. 2002.

SAMPAIO, A.B.; SCARIOT, A. Edge effect on tree diversity, composition and structure in a deciduous dry forest in Central Brazil. Revista Árvore, Viçosa, v. 35, n. 5, p. 1121-1134, set./out. 2011.

SCARIOT, A. Forest fragmentation effects on palm diversity in central Amazonia. Journal of Ecology, v. 87, n. 1, p. 66-76. 1999.

SCHEER, M. B. et al. Estrutura arbórea da Floresta Ombrófila Densa Altomontana de serras do Sul do
Brasil. Acta Botânica Brasilica, Feira de Santana, v. 25, n. 4, p. 735-750, out./dez. 2011.

SEGER, C. et al. Levantamento florístico e análise fitossociológica de um remanescente de Floresta Ombrófila Mista localizado no município de Pinhais, Paraná-Brasil. Floresta, Curitiba, v. 35, n. 2, p. 291-302, mai./ago. 2005.

SILVA, A. C. et al. Relações florísticas e fitossociologia de uma Floresta Ombrófila Mista Montana secundária em Lages, Santa Catarina. Ciência Florestal, Santa Maria, v. 22, n. 1, p. 193-206, jan./mar. 2012.

SWAINE, M. D.; WHITMORE, T. C. On the definition of ecological species groups in tropical rain forest. Vegetatio, v. 75, p. 81-86. 1988.

TABARELLI, M. et al. Forest fragmentation, synergisms and the impoverishment of neotropical forests. Biodiversity and Conservation, v. 13, p. 1419-1425. 2004.

VIANA, V. M.; PINHEIRO, L. A. F. V. Conservação da biodiversidade em fragmentos florestais. Série técnica IPEF, v. 12, n. 32, p. 25-42, dez. 1998.

VIBRANS, A. C. et al. Structure of mixed ombrophyllous forests with Araucaria angustifolia (Araucariaceae) under external stress in Southern Brazil. Revista de Biologia Tropical, San José, v. 59, n. 3, set. p. 1371-1387. 2011.

WILLIAMS-LINERA, G. et al. Microenvironment and floristics of different edges in a fragmented tropical rainforest. Conservation Biology, Boston, v. 12, n. 5, p. 1091- 1102. 1998.

WOOD, S. N. Thin plate regression splines. Journal of the Royal Statistical Society: Series B (Statistical Methodology), Hoboken, v. 65, n. 1, p. 95-114, 2003. 